\title{
Scientific publication and preprint
}

\begin{abstract}
Open access preprint publications are growing in number. This new context of science dissemination poses deep challenges for scientific publications. This paper seeks to bring the reader and the researcher to the context of the policy followed with regard to preprint publications by the Sociology International Journal as an international open access peer-reviewed journal.
\end{abstract}

Keywords: preprint, scientific, publication, scientific dissemination, sociology, international journal
Volume 3 Issue 2 - 2019

\author{
Sandro Serpa \\ Department of sociology, University of the Azores, Portugal
}

Correspondence: Sandro Serpa, University of the Azores, Faculty of Social and Human Sciences, Department of Sociology, Interdisciplinary Centre of Social Sciences - CICS.UAc/ CICS.NOVA.UAc, Interdisciplinary Centre for Childhood and Adolescence-NICA-UAc, Ponta Delgada, Portugal,Tel 351964424554,Email sandro.nf.serpa@uac.pt

Received: March 25, 2019 | Published: March 27, 2019

\section{Sociology international journal and preprint publications}

With the Sociology International Journal (SIJ) consolidating itself in the academic world, ${ }^{1}$ it is necessary to look into the several challenges that arise. In this paper, the specific situation of the preprint, which, in one of my previous editorials, was already presented as one of the challenges to be successfully tackled: "the emergence of the preprint publication, with a growing acceptance in the social sciences field, with its potential advantages and limitations"(p. 102). ${ }^{1}$ This is the topic that I will develop next.

Open access preprint publications are currently increasing in number in the context of scientific publishing, and the trend points, from our standpoint, towards their increase, even in scientific disciplinary areas where there is no such tradition, such as social sciences. $^{2}$

On the basis of a previous publication, ${ }^{2}$ it may be asserted that there are several platforms where it is possible to publish online academic manuscripts in the preprint format, usually in an open access system. In Elmore's ${ }^{3}$ excellent perspective, "The traditional definition of a preprint is a document that has been uploaded to a preprint server, is freely accessible to the public, and has not previously been published in a journal. Posting generally occurs the day of submission or the next day. There is typically no formal peer review of the article before it is posted online; however, it is checked for plagiarism and offensive/ dangerous content. The only requirement is that the article is scientific in nature. There is no typesetting or editing before posting online. Because they are not journals, preprint servers have no impact factor and authors retain copyright of their articles. Each online version of an article allows comments to be posted by the public" (p. 364).

The preprint format entails that manuscripts are published very quickly, in most situations after a first summary assessment that mainly validates the manuscript's structure and ethical aspects. In the preprint model, manuscripts are not assessed according to the stricter scrutiny of a traditional peer-review process, usually before a possible publication, in any of its several possible modalities. For example, double-blind review (reviewers and authors remain anonymous among themselves); blind-review (with the reviewer's identification but the author's anonymity), single-blind review (with the reviewer's anonymity and the author's identification), or in the form of openreview (both reviewers and authors are identified).

In the scientific publication based on peer-review, this process functions as control of the manuscript's scientific quality. According to Ferreira \& Serpa, "Through the analysis, review, and critique of the work by their peers, scientists aim to ensure that only research that is part of research programs legitimised by the respective scientific community is published and recognised" (p. 12). This process allows "a priori control through the evaluation of the article by peer specialists in the field, which legitimises the editorial decision whether to publish it or not" (p. 13), as well as the resulting legitimacy and credibility of what is published and of the scientific journals themselves.

Returning to the preprint format, besides the speed of publication, this process makes available to the reader manuscripts that have at least a minimum scientific quality, ascribing the readers the responsibility to assess the quality and select the manuscripts according to their interest and knowledge. This possibility of publishing in preprint or draft versions, which offers the opportunity that the readers' comments may enhance the manuscript in a later version, calls into question an academic principle of social sciences: Those scientific journals would accept, almost exclusively, for review and possible publication, only unpublished manuscripts. There is an increasing number of scientific journals accepting the submission and consequent review of enhanced versions of manuscripts published in an earlier preprint version. It is, of course, required that the corresponding author informs the publisher that previous versions of the manuscript are published.

This whole new context of preprint scientific publications, with their advantages and limitations (for further development on this topic, see, ${ }^{2}$ poses challenges for the Sociology International Journal (SIJ). SIJ is geared to the publication of several types of papers "by social scientists from around the world, providing insight into the issues facing by the society as well as social and cultural systems in other countries", 5 and Sociology functions as the main driving force of the journal.

Considering the potential advantages of the preprint logic, SIJ accepts the submission of original manuscripts, as well as manuscripts that may have been published in a preprint format (i.e., that have not gone through an exhaustive peer-review process), provided that the corresponding author informs the publisher of that/those previous publication(s), as well as the corresponding bibliographic reference. As Editor-in-Chief, I reinforce SIJ's goal of publishing manuscripts of various types with a focus on Sociology as a multi-paradigmatic Science, ${ }^{6}$ but always on the respect of the values of quality and democracy $^{7}$ in the scientific publication.

\section{Acknowledgments}

I would like to thank to the Editor and the Reviewers for their comments and suggestions. 


\section{Funding}

University of the Azores, Interdisciplinary Centre of Social Sciences-CICS.UAc/CICS.NOVA.UAc, UID/SOC/04647/2013, with the financial support of the FCT/MEC through national funds and when applicable co-financing from the FEDER under the PT2020 Partnership Agreement."

\section{Conflicts of interest}

The author Sandro Serpa is advisory board member of Preprints, a platform that publishes versions of papers without peer review.

\section{References}

1. Serpa S. Consolidation and challenges. Sociol Int J. 2019;3(1):102-103.
2. Ferreira CM, Serpa S. The importance of preprint in scientific publication: Perspectives and challenges. The Journal of Social Sciences Research. 2018a;4(12);642-647.

3. Elmore SA. Preprints, what role do these have in communicating scientific results? Toxicologic Pathology. 2018;46(4):364-365.

4. Ferreira CM, Serpa S. Publicising the identified peer-reviewer: Legitimacy and quality of the scientific publication. The International Journal of Interdisciplinary Educational Studies. 2018b;13(1):11-17.

5. Sociology International Journal. Aim and Scope. Accessed on March 20, 2019.

6. Serpa S. Reflexivity in Sociology. Sociol Int J. 2018a;2(6):584.

7. Serpa S. Quality and democraticity in scientific dissemination. Sociol Int J. 2018b;2(5):429. 